Convergences francophones 6.3 (2020): 16-28

http://mrujs.mtroyal.ca/index.php/cf/index

\title{
Penser au-delà du binarisme: Étude des stratégies de reconnaissance et de ré/conciliation des altérités dans Aux États-Unis d'Afrique d'Abdourahman Waberi
}

Jennifer Boum Make Georgetown University, États-Unis

Dans le roman d'Abdourahman Waberi, Aux États-Unis d'Afrique (2006), la zone géographique euraméricaine devient le miroir inversé d'une Afrique fictive présentée comme le centre économique, politique et culturel du monde. Dans cette logique d'inversion, le continent africain alors renommé les États-Unis d'Afrique établit une rupture physique et idéologique avec l'Euramérique : les 'infortunées terres d'Euramérique' sont construites en opposition avec la fédération prospère des États-Unis d'Afrique qui garde farouchement ses frontières contre ces Autres fantasmés mais avant tout diabolisés, « ces peuplades aux mœurs guerrières, aux coutumes barbares, aux gestes fourbes et incontrôlables » (12). Le recours à des oppositions binaires pour incarner les rapports entre États-Unis d'Afrique et Euramérique entretient une rhétorique de l'altérité des frontières physiques et mentales entre les Uns et les Autres. Ceci n'est pas sans rappeler le modèle eurocentrique colonial incarné par l'image du 'miroir d'Hérodote'. ${ }^{1}$ Décrivant la rhétorique de l'altérité coloniale, Anthony Mangeon précise que le jeu de miroir suppose ici une «opposition entre le monde européen civilisé, intelligent, élevé dans la véritable religion ... et le monde noir, ignorant, superstitieux et plus proche des bêtes que des hommes » (22).

En adoptant le schéma binaire sur lequel reposent les rapports de force asymétriques caractéristiques de l'altérité coloniale, la dialectique du dominant et du dominé opposant les États-Unis d'Afrique, représentant le monde civilisé, et l'Euramérique la barbarie, permet non seulement la récupération et le travestissement de références culturelles propres au monde occidental, ${ }^{2}$ mais aussi une interrogation sur un modèle d'opposition binaire. Ainsi l'hypothèse soutenant ce travail tend à remettre en question un modèle d'opposition binaire symptomatique des systèmes de représentation coloniaux pour donner à voir de possibles voies de ré/conciliation 'des particularismes locaux' ${ }^{3}$ au-delà des dichotomies dominant/dominé. Il s'agit ainsi de s'interroger sur les conditions

\footnotetext{
${ }^{1}$ Dans La pensée noire et l'Occident: de la bibliothèque coloniale à Barack Obama (2010), Anthony Mangeon s'intéresse à la manière dont les écrits d'Hérodote ont permis aux Grecs de construire leur identité par opposition aux représentations du monde non-grec. Le miroir d'Hérodote agit donc d'abord comme « un 'miroir en négatif' du monde grec » (17).

2 Sauf indication contraire, toute mention du 'monde occidental' dans ce travail se réfère à la construction et diffusion d'une aire culturelle et historique occidentale monolithique comme théorisée dans les travaux d'Edward Saïd notamment Culture and Imperialism (1993). La démarcation nette entre un monde occidental civilisé et le reste contribue ensuite grandement à justifier l'impérialisme culturel de l'Occident.

${ }^{3}$ Voir le plaidoyer d'Anthony Appiah pour ce qu'il appelle 'un cosmopolitisme ancré' visant à la survivance et la coexistence des particularismes locaux dans Pour un nouveau cosmopolitisme (Odile Jacob, 2008).
} 
permettant de travailler à l'instauration de formes d'interaction sociale et culturelle tout en signalant un espace de réflexion éthique et esthétique qui succède à la chute des rapports de domination culturelle, politique et économique dans l'œuvre de Waberi.

On se propose donc dans un premier temps de remettre en question les limites du modèle d'opposition binaire en problématisant les conditions de son émergence dans la fiction et ses effets. Il s'agira dans un second temps de considérer la mise en texte et la dissémination de possibles voies de ré/conciliation des altérités comme tactique de subversion des rapports de domination ayant cours dans le roman.

La mise en place d'un patrimoine culturel africain suggère tout d'abord le dépouillement des biens matériels et immatériels des anciennes puissances économiques euraméricaines, et engage ensuite une pratique conjointe de destitution et d'attribution systématique de noms 'africanisés,' c'est-à-dire inscrits spatialement et culturellement dans les États-Unis d'Afrique. En effet, Waberi pose dès les premières pages le décor d'un monde imaginé et propose une doublefictionnalisation: une fictionnalisation par le processus de création romanesque ainsi qu'une fictionnalisation géographique et culturelle. Le Nord de l'envers et les États-Unis d'Afrique de l'endroit (pour reprendre la métaphore de la territorialité japonaise $)^{4}$ sont non seulement les vecteurs de rapports de domination mais tracent plus précisément les contours d'un binarisme: le Sud géographique impose l'hégémonie africaine au reste du monde. Au cour de cet espace mondial redessiné, Maya/Malaïka (prénom d'adoption), personnage central du récit, est en mouvement : quittant les États-Unis d'Afrique en quête de ses origines, elle se met en route vers le Nord, et prend le chemin du re(/dé)tour dans les dernières pages du récit. Ne pourrait-on donc pas comprendre ce voyage comme une possible subversion du modèle d'opposition binaire en ce que le personnage de Maya/Malaïka perturbe la vision de l'Autre comme altérité radicale et irréductible par le biais de sa circulation, son errance entre deux espaces au départ fermement antagonistes? Ainsi, en quoi peut-on déceler dans l'œuvre de Waberi plusieurs niveaux de déstabilisation du modèle d'opposition binaire sous-tendant une miseà-distance couplée à l'assujettissement de l'Autre, qui atteignent leur culmination avec le motif de l'errance ? Dans quelles mesures, le voyage de Maya met-il en garde contre l'assignation d'identités culturelles et géographiques stables et essentialisées, et travaille enfin à son dépassement ?

\section{Interroger le binarisme colonial/postcolonial}

Comme nous le rappelle Mangeon, il existe, dans le champ des littératures coloniales (ou 'bibliothèque coloniale') un paradigme prévalant qui repose sur un binarisme colonial fondateur et, plus précisément, l'expression d'une domination

\footnotetext{
${ }^{4}$ Dans un imaginaire économique suranné et essentialisant, la construction de l'espace japonais suggérait une division géographique virtuelle sur des bases économiques : le Japon de l'endroit d'une part (forte concentration humaine et production industrielle intense), et le Japon de l'envers d'autre part (relativement moins peuplé et faible développement économique).
} 
culturelle, économique, et sociale sur les peuples colonisés. En contrepoint des discours coloniaux, les littératures postcoloniales africaines sont une manifestation des formes de questionnement de l'épistémologie occidentale et notamment du canon littéraire européen. ${ }^{5}$ Dans l'introduction de On the Postcolony (2001), Achille Mbembe s'intéresse notamment à deux modalités dans le rapport entre ces Uns et ces Autres accompagnant dans certains cas le 'tournant postcolonial'. Il distingue d'une part une représentation essentialiste et infériorisante d'une Afrique tenue à distance, caractéristique chez les artisans de la bibliothèque coloniale, à savoir : "Africa, a headless figure threatened with madness and quite innocent of any notion of center, hierarchy, or stability, is portrayed as a vast dark cave where every benchmark and distinction gone together in total confusion" (3). D'autre part, Mbembe distingue dans le discours afrocentrique en particulier l'expression d'un 'African self' logocentrique et finalement lui aussi essentialisé. Commentant sa remarque sur la polarisation du discours européen et africain, Mbembe donne voix à une impasse : celle d'une écriture cyclique et stérile, mettant en opposition constante 'the traditional African life' et 'the absoluteness of the African self' en ce que "both [assert] denial and the reaffirmation of that humanity [that of the 'African self'] now look like the two sterile sides of the same coin" (12).

Différemment, lire le texte de Waberi dans une recherche de ré/conciliation des différences entre ces Autres antagonisés s'élabore en quelque sorte en réponse aux propos de l'auteur lors de son entretien avec Alain Mabanckou : ce dernier évoquait notamment le refaçonnement des frontières géographiques comme « une farce subtile ou grossière » (9). La mise en texte de cette 'farce' survient dans les premières pages du récit et constitue à bien des égards un renouvellement de l'imaginaire géographique d'une part, et des représentations idéologiques et identitaires d'autre part. Les potentiels du geste artistique opéré par Waberi apparaissent à deux niveaux distincts : l'écrivain donne tout d'abord corps à une inversion du binarisme colonial, permettant de s'interroger sur les conditions d'émergence de ce modèle de domination et ses limites, et c'est ensuite au sein de ce renversement que se situe un espace de réflexion éthique et esthétique où penser les particularismes culturels en dehors du modèle binaire dominant/dominé.

Pour Edward Saïd, les dominations coloniales reposent sur le schéma axiologique du 'Nous-contre-Eux [Us vs. Them]' ${ }^{6}$ là où la conception essentialiste de 'l'Orient' par 'l'Occident' pose l'autorité discursive et le contrôle des représentations de l'Un sur l'Autre, l'ailleurs oriental, ou plutôt le monde nonoccidental. Dans le roman de Waberi, la stratégie d'inversion s'attache

\footnotetext{
${ }^{5}$ Pour un passage en revue des formes de mise en question de l'épistémologie occidentale dans les littératures africaines, par comparaison, confrontation ou simple souci de pluralisme épistémologique, voir « Philosophie en Afrique » dans La pensée noire et l'Occident, pp. 97-194. Pour une articulation critique du tournant littéraire et politique postcolonial, voir en particulier Ashcroft, Bill, Gareth Griffiths et Helen Tiffin. The Empire Writes Back: Theory and Practice in Post-Colonial Literatures (1989).

${ }^{6}$ Pour une analyse de l'élaboration du système binaire entre les constructions que sont l'Occident et l'Orient, ou 'Nous-contre-Eux,' voir Orientalism (1978), notamment l'Introduction et le Chapitre 1, "The Scope of Orientalism."
} 
particulièrement à une manipulation en miroir de la dialectique des pronoms. Le 'je' du narrateur, qui demeure anonyme, établit précisément ce rapport d'opposition entre un 'nous' de proximité et un 'ils' lointain qui désigne 'les immigrés extraafricains,' les nouvellement nommés Euraméricains (15).

C'est dans ce geste de réattribution sémantique que Waberi construit les États-Unis d'Afrique comme autorité écrasante d'où vont émaner des systèmes de représentation servant à l'élaboration conceptuelle d'une altérité euraméricaine radicale ainsi que des normes discursives et socioculturelles monologiques. Remarquons par exemple les inflexions du langage lorsque le narrateur reprend l'expression populaire 'un autre son de cloche,' à laquelle il préfère 'un autre son de minaret'(36), ou encore lorsque ce dernier renomme le prix Nobel de la paix, le prix Arafat (16), par le biais d'une ré-appropriation des références culturelles en circulation dans le monde occidental. La liste de ces altérations est longue, et il ne s'agit pas d'en faire l'inventaire mais plutôt de décomposer les mécanismes d'une assise culturelle et idéologique par le biais de manipulations linguistiques et surtout sémantiques.

La stratégie de l'inversion chez Waberi soutient une esthétique fictionnelle qui donne à voir une réalité autre, un monde alternatif possible, ${ }^{7}$ faisant ressortir les fondements d'une matrice idéologique. Notons par exemple l'usage du référent 'Tiers-Monde' qui désigne à présent le Nord appauvri. Au-delà de suggérer une simple rupture provisoire entre signifiant et signifié dans la mesure où le 'TiersMonde' indique communément les pays situés dans l'hémisphère Sud prétendument dépourvus des atouts économiques faisant loi dans le monde occidental, ce remaniement sémantique concerne l'arbitraire et la relativité de tout système linguistique se posant comme autorité. Il dévoile les rouages d'une tactique d'essentialisation en ce qu'apposer le référent 'Tiers-Monde' au Nord géographique détermine les conditions d'existence de celui-ci.

Le renversement géographique et idéologique du monde porté par l'accumulation de manipulations discursives comme celles évoquées plus tôt va de pair avec un exotisme ainsi qu'un processus d'exotisation à l'envers. Le corps de l'immigré extra-africain est alors exotisé, ou devient exotique. La mise en scène et les éléments de description de ces figures exotiques au demeurant anonymes se réduisent à leurs «tiges d'albâtre aux pommettes rose de lingerie et aux fesses plates » (72). Ici, l'exotisation s'apparente à une pratique discursive monologique : celle-ci isole l'Autre et en produit une représentation totalisante, objectivante et stéréotypée. Remarquons enfin que la description du corps de l'Autre suggère une

\footnotetext{
${ }^{7}$ Dans Fondements et représentations identitaires chez Ahmadou Kourouma, Tahar Ben Jellou et Abdourahman Waberi (2009), Cheikh Mouhamadou Diop s'intéresse à l'œuvre de Waberi (entre autres) pour démontrer les potentiels de la fiction contemporaine dans la confrontation de visions alternatives possibles à un modèle idéologique dominant (25). D'ailleurs, Waberi soutient l'éclatement d'une écriture fictionnelle circonscrite lorsqu'il défend une fuite de l'aura culturelle française qu'il considère à bien des égards fossilisée, « tournée vers cet hier chatoyant » (« Écrivains en position d'entraver » dans Pour une littérature-monde (2007: 72). Voir aussi La pluralité des mondes : vers une nouvelle anthropologie de Francis Affergan (1997).
} 
fragmentation de ce dernier, une représentation métonymique qui en identifiant les marqueurs d'exotisme en dévoile le processus de formation de l'être exotique.

Précisons qu'attribuer une dimension monologique à la construction du corps exotique structure la représentation de l'Autre et marque une certaine emprise discursive du 'je' de l'énonciation ainsi que du 'nous' auquel il se rattache. Cette assignation du corps exotique depuis les États-Unis d'Afrique s'inscrit dans la poursuite d'un raisonnement binaire, cette fois afrocentrique plutôt qu'eurocentrique. Dans son étude de ce qu'il considère comme le versant concurrentiel de la bibliothèque coloniale, Mangeon conçoit notamment 'l'afrocentrisme' dans des termes similaires aux discours de la bibliothèque coloniale, c'est-à-dire comme un « essentialisme culturaliste » (175). Il suggère, «... l'afrocentrisme finit toujours par trahir son eurocentrisme déguisé, tandis que sous couvert d'opérer une révolution philosophique, il n'offre rien d'autre qu'une réation à tous les sens du terme » (182). À l'inverse, Diop envisage cette logique afrocentrique dans une compréhension utopiste du renversement des oppositions binaires en ce qu'elle participe d'un « fantasme d'hérö̈sation » alors associé à « des formes de galvanisation » qui contiennent «le secret de pérennité des peuples et des imaginaires » (19). Dans $A u x$ É-U d'Afrique, le narrateur annonce en particulier : "Plus tard, le Gondwana se disloquera en moult continents dérivant, mais seule l'Afrique restera fixe, au centre du monde. Tu retiendras l'essentiel : l'Afrique était déjà au centre et elle le reste encore » (55). Est-ce là bien ce dont il s'agit?

\section{La déconstruction/mise en échec? de structures d'enfermement}

Si le processus de fictionnalisation soutient de prime abord le renversement du monde par un entrelacs de manipulations discursives, la présence de signes d'une remise en question de ce geste esthétique pose question. Waberi suggère que la production du substrat identitaire se situe dans les objets matériels qui nous environnent et leur inscription dans un système de référentialité donné. Dans le récit, cela donne lieu à une réassignation des instances de production d'une altérité infériorisée, essentialisée et mise à la marge: les États-Unis d'Afrique s'approprient une autorité discursive et idéologique, auparavant monopole exclusif du monde occidental. Une fois le binarisme renversé et dans le prolongement de la réflexion engagée par Aedín Ní Loingsigh dans son article "Travel and Language in Aux États-Unis d'Afrique", il s'agit maintenant de questionner les potentiels d'une déstabilisation du modèle d'oppositions binaires. Loingsigh soutient notamment que "Waberi [also] suggests in the novel that although political and economic realities conspire to present the world as divided, stories and fiction have the power to challenge this geography by circulation beyond border" (79). Se dessine alors au niveau métatextuel une possible voie de sortie du binarisme.

La dimension métatextuelle du récit réside précisément dans la possibilité de mettre en échec le renversement du monde, et dans une plus large mesure, toute structure binaire en ce qu'elle serait irrémédiablement engagée dans un rapport de

mise à distance de l'Autre. Dans Aux É-Ud'Afrique, c'est sur un ton apparemment 
ironique que repose la construction d'un monde en contre-point en ce que poser le cadre de référentialité de celui-ci consiste à engager un travestissement parodique du patrimoine culturel et historique des 'immigrés extra-africains'. Pour ne citer que quelques exemples parmi tant d'autres : le spationaute Neil Armstrong est renommé Ezra Mapanaza (29) ou encore, L'Origine du Monde aurait été peinte par Gustavo Mbembe et non Gustave Courbet (104). Tandis que certains critiques, comme Gloria Nne Onyeoziri dans Shaken Wisdom (2011), soutiennent que dans les fictions africaines postcoloniales, l'ironie est un outil essentiel au détournement des imaginaires attachés à une Afrique tant fantasmée qu'essentialisée, ${ }^{8}$ c'est le recours à l'ironie qui génère chez Waberi ce qu'on appellerait une 'dystopie inversée' en ce que le processus d'africanisation n'offre aucune voie de sortie du modèle logocentrique au sein duquel l'Autre est cet éternel marginalisé. Tout comme le suggère Anjali Prabhu dans son analyse du schéma d'oppositions binaires, " l'Afrique du roman de Waberi n'est guère meilleure que l'Europe, ni plus rachetable que l'Euro-Amérique dont elle reproduit les crimes et les jeux d'exclusion » (83).

Ainsi, le potentiel subversif de l'ironie est étouffé par les nouvelles structures d'enfermement qu'il provoque dans le récit; à savoir une reproduction en miroir des travers du rapport dominant-dominé dans les domaines politique, culturel et économique. Ce premier niveau d'ironie est démonté/s'effondre ? lorsque le monde inversé devient lui-même cible d'une nouvelle ironie anticipant sa mise en échec. Le monde renversé, ou inversé donne lieu à une fermeture spatiale et enferme les imaginaires de l'altérité. Les États-Unis d'Afrique sont conçus comme un espace clos, aux frontières imperméables freinant l'accès des 'immigrés extraafricains' et prônant une politique d'inhospitalité. En s'inscrivant à nouveau dans le prolongement d'une inversion en miroir, les É-U d'Afrique reproduisent des pratiques apparues inhospitalières en Europe occidentale. Citons par exemple l'état des lieux de Tahar Ben Jelloun au sujet de l'appareil étatique français qu'il juge complice de l'inhospitalité : "L'hospitalité française est ainsi ruinée, rendue difficile, voire impossible. C'est l'époque du malheur balbutiant. Plus de place, plus de temps pour la gratuité du geste, pour comprendre, accepter celui-là au regard hésitant, venu d'une autre durée » (14).

À l'image du rétrécissement spatial évoqué par Ben Jelloun dans Hospitalité Française (1983), la disparition, si ce n'est l'absence de voie vers l'Autre, se manifeste dans Aux É-Ud'Afrique au travers d'une écriture de l'enfermement, un enfermement tout d'abord spatial qui prend forme dans la matérialité des frontières : « Nous déjà à vos portes, à vos barricades, vos barrières, vos murs parpaings. Vous déjà calfeutrés dans les villes, dans les gares, les aéroports, les jardins publics, et un bruit d'apocalypse nous précédant à chaque pas » (98). Notons d'ailleurs que c'est la seule occurrence d'une voix narrative propre aux 'immigrants extra-africains' avant le départ de Maya pour Paris. C'est une voix qui appelle à la

${ }^{8}$ Onyeoziri défend que dans les fictions postcoloniales africaines, "the trope of irony lends itself to the opening up, destabilization and deconstruction of the imaginary essences, doxa and fixed categories that have defined and continue to define the relationship between Africans and other peoples" (3). 
solidarité de la 'grande Afrique,' mais qui révèle aussi le cannibalisme de ses lieux (in)hospitaliers (97). C'est une voix qui, tout bien considéré, est contenue entre les lignes d'une lettre retrouvée par la police maritime de Port-Soudan dans les poches d'un anonyme ; elle-même absorbée par la voix narrative d'un 'je' omniprésent, une voix finalement bien calfeutrée et étouffée au sein même de la construction du récit. L'impasse spatiale du migrant, conditionnant l'ambivalence de sa voix, est symptomatique d'une impasse de l'écriture, celle du binarisme. La mise en échec du geste esthétique dépositaire de la création des États-Unis d'Afrique sous-tend donc le refus d'une simple africanisation des cadres de référentialité du monde occidental, qui n'élabore dans la fiction qu'une dystopie en contre-point.

Ainsi, le potentiel utopique, ou du moins émancipatoire, réside plutôt dans l'incitation à échapper au binarisme et à l'univers totalisant de la 'grosse Africa' (98). Il s'agit à présent d'interroger les stratégies de ré/conciliation des différences culturelles et les enjeux éthiques et esthétiques d'une sortie de l'écriture du binarisme. Ainsi, à quoi s'apparentent les marqueurs d'une écriture de l'ouverture? Quelles stratégies de ré/concialition le texte de Waberi donne-t-il à voir?

\section{Exploration des potentiels du nomadisme et de l'errance}

Chez Waberi, les 'immigrés extra-africains' sont les hominis viator dont l'écrivain fait l'éloge dans son «Éloge du Nomadisme » (2005). Il reconnaît la nécessité économique qui pousse les nomades à s'engager sur la route, mais il révèle déjà dans leur mode d'existence en élision, la possibilité d'une ouverture à l'Autre et ses particularismes. Selon Waberi, «Le nomade est déjà loin ... . Difficile de le mettre en mots .... Ne reste plus qu'à se faire libre-rêveur à défaut de le suivre à la trace sur les routes, les chemins buissonniers, les lacets qui montent et descendent à bout de souffle .... Idem pour nous diasporisés et natifs-natals réunis » (132). L'écrivain permet d'envisager l'immigré-nomade comme la figuration d'une mise en contact des particularismes par son éparpillement dans l'espace. Waberi renouvelle d'ailleurs son attachement à la notion de 'nomadisme' qu'il juxtapose à un 'désir d'ailleurs' lorsqu'il révèle à Mabanckou : « Aux ÉtatsUnis d'Afrique n'est que la poursuite logique de ce mouvement nomadique. Je suis un nomade de tempérament. »"

Dans le récit, les immigrés s'engagent dans un mouvement nomadique. Ils se heurtent certes aux frontières Sud mais en appellent toutefois à un mouvement vers un ailleurs. Confrontée à la vue de ces derniers, Maya est tout d'abord interpellée : ils pénètrent dans le domaine du visible et s'imposent d'une certaine façon à son regard (82). Ce moment de rencontre entre l'Un et l'Autre permet de procéder à une première sensibilisation aux différences observées chez l'Un et l'Autre. Tandis que les 'immigrés extra-africains' s'exclament : « Halte à l'hégémonie africaine » ou encore, « Un autre monde est possible, » le narrateur ajoute à l'adresse de Maya : « Et tu les as rejoints, Maya ! ... Mais as-tu compris un traître mot à leurs discours intergalactiques, leur envie de conjuguer le proche et le lointain, leur fumeuse alchimie entre l'universel et le local » (84). L'exclusion de l'Autre prônée par les 
É-U d'Afrique est dès lors déjà perturbée par une tentative de sensibilisation à une pensée de la différence.

Plus précisément, celle-ci s'accompagne d'un éclatement de la voix narrative qui incorpore d'abord la parole de l'immigré pour la première fois dans le passage cité, et atteste ensuite de l'existence du 'tu,' de l'Autre. Le 'tu' se place au sein d'une masse solidaire : l'inscription de cette voix pronominale de l'entre-deux, située à mi-chemin entre le 'nous' des États-Uniens d'Afrique et le 'ils' des Euraméricains permet la mise en relation des particularismes. La définition par Édouard Glissant du terme 'Relation' engage une 'racine démultipliée'. Dans Poétique de la Relation (1990), il y accole 'la pensée du rhizome,' qu'il définit comme « un principe de ce qu' [il] appelle une poétique de la Relation, selon laquelle toute identité prétend d'un rapport à l'Autre » (23). Ladite pensée de l'autre, ce 'rapport à l'Autre' qu'évoque Glissant, se reflète dans l'existence même du personnage de Maya/Malaïka. Cette enfant unique adoptée par Docteur Papa, travailleur humanitaire, a quitté la France et vit depuis sur le continent africain. Dès l'apparition du personnage dans le récit, c'est un questionnement perpétuel qui prend forme et s'articule autour de la question qui lui est posée indirectement : «Qui suis-je, te demandes-tu parfois, Maya ?» (63).

\section{La reconnaissance d'une insaisissable diversité comme acceptation de soi et de l'Autre}

Maya est certes celle qui perçoit la présence de ces Autres physiques, à savoir les 'immigrés extra-africains,' mais son rapport à l'Autre consiste tout d'abord à reconnaître la présence d'une altérité, d'une insaisissable diversité en elle. « Nous vivons désormais en présence de tous les habitants du monde. Cette présence aux autres passe cependant par la présence à soi, » déclare notamment Glissant dans Poétique (122). L'analyse de l'articulation d'un travail sur le rapport à soi donne à voir une possible ré/conciliation des différences jusqu'alors antagonisantes.

Plusieurs éléments textuels participent à concevoir Maya comme figure en mouvement. La jeune fille atteste d'une variation des pratiques discursives en ce qu'elle se démarque de la pratique conjointe de destitution/restitution du nom engagée dans le récit afin d'asseoir la vision totalisante des États-Unis d'Afrique. La double dénomination Maya/Malaïka résiste à une pétrification linguistique et sémantique. Elle est celle que le narrateur définit comme «une jeune fille pas ordinaire. Une jeune fille qui refusera de répondre à la sonnette du nom propre » (20). En effet, il n'est question à aucun moment dans le récit de renoncer à Maya pour Malaïka, ou vice versa. Bien au contraire, le récit est porté par le va-et-vient d'un nom propre à un autre. Fixer l'usage du nom propre, c'est déjà modeler une identité référentielle stable. L'instabilité des pratiques discursives génère d'une part l'inadéquation du nom propre, et d'autre part l'acceptation d'une diversité dans la présence à soi, et dans la présence au monde, qui vient plus tard admettre l'existence du 'tu' dans le récit.

De plus, Maya apparaît comme le véhicule d'une diversité insaisissable, traduite par sa quête certes vaine des origines, mais toutefois garante d'une prise de 
contact sans cesse renouvelée avec l'inconnu. Waberi propose par l'entremise de ce personnage un éparpillement spatial qui donne à voir un processus de décentrement, un détachement de l'univers totalisant des É-U d'Afrique. Enfin, Maya serait une manière d'inaugurer le concept de 'littérature-monde' dont l'écrivain se fait le porte-parole dans le manifeste littéraire du même nom, et qui rassemble « une génération d'écrivains issus de l'émigration, [qui] au lieu de se couler dans sa culture d'adoption, entendait faire œuvre à partir du constat de son identité plurielle, dans le territoire ambigu et mouvant de ce frottement » (Onyeoziri n.p.). C'est bien ce même constat qui nourrit le principe d'instabilité signalé par le personnage de Maya : à savoir l'hésitation dans l'attribution définitive d'un nom propre ; le mouvement spatial depuis la fédération africaine jusqu'à l'hémisphère Nord ; et finalement, l'enchevêtrement des temporalités entre le passé opaque de Maya et un présent qui résiste toute totalisation spatio-temporelle. Le recours à ces trois éléments participe à la mise en texte progressive de l'errance et engage un processus d'ouverture à l'Autre.

La quête des origines dans un premier temps et la tentative de retour à ces mêmes origines dans un second temps sont des forces qui catalysent l'errance de Maya. Le personnage amorce son parcours nomade dans le souci d'atteindre une présence à soi dont l'aboutissement serait le dévoilement de ses origines. Ce parcours initial se mue ensuite en un apprentissage de l'errance face au mouvement renouvelé du personnage dans des espaces autres, alors ponctué d'interactions avec les particularismes de ceux et celles dont elle croise la route. Le mouvement de Maya est tout d'abord métaphorique : sa transformation symbolique en oiseau permet d'obtenir un corps transfiguré par l'errance. Ainsi, " prisonnière d'aucune frontière. Oiseau des routes du ciel, aimanté par l'envol, attiré par les voyages, les haltes, les intermittences et les vols prodigieux, » l'existence métaphorique de la jeune fille laisse entrevoir un premier niveau d'éparpillement spatial (49). Cela change son rapport au corps ainsi que celui qu'elle entretient avec le lieu. Les frontières des É-U d'Afrique sont bafouées, rendues caduques, si ce n'est même décomposées. En d'autres termes, ce premier envol métaphorique est placé sous le signe de la rupture, mais une rupture productrice qui associe étroitement refaçonnement géographique et identité.

C'est dans le contact avec l'inconnu du lieu que se développe la valeur initiatique de l'errance de Maya. La rencontre directe du personnage avec l'inconnu d'un espace autre alimente un apprentissage par la répétition. La récurrence d'une rencontre avec l'altérité participe pour Maya à la connaissance de particularismes locaux dépassant le projet originel d'une quête des origines. Après le déploiement métaphorique de son existence, penser l'errance de Maya passe par la mise en scène d'un lieu de rêverie, un espace d'invention se tenant au point de rencontre entre soi et les autres, dépositaire d'une esthétique et éthique d'ouverture à l'altérité. En particulier, Maya s'adonne à la sculpture et se livre au cœur de son atelier à « un commerce sensoriel entre ce territoire et le reste du monde » (108). L'activité artistique du personnage s'inscrit dans le prolongement de sa transfiguration métaphorique car celle-ci suggère de profondes interactions avec un ailleurs 
fantasmé provoquant la rencontre d'un monde connu jusque-là circonscrit et celui habité par d'autres.

L'atelier de Maya devient un lieu emboîté de la création fictionnelle/artistique. Se tenant en marge de l'espace totalisant de la fédération africaine, Maya seule dans son atelier bricole la diversité de son existence, s'abandonnant à des imaginaires qui la conduisent au carrefour des mondes. Notons aussi que c'est railler une nouvelle fois le binarisme que d'encloisonner Maya dans son atelier, car c'est « devant [son] jardin, ou plus exactement devant [son] atelier, [qu']on se retrouve face à un nouveau monde. Un lieu de rêverie, d'imagination, de fiction » (105). Il s'agit donc d'un enfermement qui n'en est pas vraiment un, ou du moins d'un enfermement qui révèle et relaie un éparpillement identitaire, à la différence du cloisonnement des É-U d'Afrique qui lui exclut la diversité et laisse se heurter à ses portes les 'immigrés extra-africains' .

C'est en écho à l'observation de Loingsigh au sujet du personnage de Maya ${ }^{9}$ qu'on peut attacher au récit une dimension auto-réflexive. Le choix de Waberi de créer le personnage de Maya constitue un acte subversif qui vise à l'éclatement interne du récit par la remise en question de la construction binaire sur laquelle repose le fondement des É-U d'Afrique. Dans le cas de Maya, la recherche d'une présence à soi envisage la sculpture comme un véhicule allant et venant de l'intérieur vers l'extérieur, et s'affirmant par-là comme lieu pour penser le rapport à l'Autre. La sculpture en appelle certes à la puissance de l'imaginaire en ce qu'elle est la métaphore d'une relation à l'altérité non-réalisée, mais suggère a fortiori une prise de contact directe avec le monde. Il s'agit d'abord pour Maya de « trouver un modèle, établir un lien de connivence avec lui, puis mettre les mains à la pâte ..., buriner, affiner, laisser reposer, sécher, passer à la cuisson...et faire couler le tout dans le bronze » (108). La pléthore d'actions successives précédant l'existence matérielle de la statue indique la recherche et le tâtonnement propres à la rencontre avec ce qui se tient à l'extérieur, ce qui demeure encore méconnu. Poser ensuite la matérialité de la sculpture et en découvrir la texture, c'est faire un pas vers l'Autre et l'acceptation de son existence.

L'atelier de Maya est donc le lieu de rencontre renouvelé des diversités car la sculpture s'inscrit dans la répétition et l'imprévisible de la création. Si l'on comprend qu' « errer [est] donc défini comme arrachement sans cesse inachevé : forme ultime que prend le voyage pour devenir 'destinerrance,' » la sculpture devient par conséquent dépositaire de l'errance métaphorique en ce qu'elle n'implique certes pas un départ physique, mais bel et bien un refus de l'enfermement (28). Elle devient lieu d'exploration de ce qui est (ou perçu) comme autre, différent. De ce fait, l'activité à laquelle se livre Maya n'est pas anodine et élabore page après page un glissement vers l'exploration de l'altérité, une prise de contact avec une diversité aussi bien propre à son identité qu'à celle des Autres. Ce

\footnotetext{
${ }^{9}$ Loingsigh suggère notamment: "The consequences of Maya's dual identity (as reflected in her double name) become crucial not just because they inform the Western reader into a critical and analytical relationship with their own privileged location, and furthermore they reflect some of the practices that Waberi sees as crucial to defining how African authors will operate within a global literary system" (85).
} 
glissement gagne progressivement en intensité dans la mesure où un autre glissement s'opère lorsque du paysage interne éclaté de Maya, on bascule finalement dans l'inscription physique de l'errance. La méconnaissance des origines de Maya génère d'abord l'absence à soi du personnage, mais permet surtout de dépasser le stade métaphorique de l'errance. La question des origines est élaborée tel un mystère qui donne lieu plus tard à la nécessité de leur dévoilement : dès son plus jeune âge, l'errance de Maya est consignée par écrit, contenue dans les pages de son petit carnet: " Tu y couches tes impressions, les haillons de tes souvenirs, tes coups de gueule, de griffes ou de blues. Au bout de ce voyage à travers les mots et les paysages, tu reviens éreintée mais gagnée par une sérénité à nulle autre pareille » (23). La pensée de l'errance portée par la recherche d'une présence à soi atteint notamment son paroxysme dans la troisième partie du récit lorsque le voyage de Maya a finalement lieu.

\section{Conclusion : l'errance comme lieu d'apprentissage du rapport à l'Autre}

Reste à questionner en quoi l'errance physique de Maya permet de dévoiler les voies d'exploration d'une reconnaissance et ré/conciliation des altérités. Abandonnée après sa naissance en Normandie par sa mère Célestine, Maya sera recueillie par Docteur Papa qui l'emmènera vivre à Asmara; le voyage de la jeune fille suggère donc qu'elle s'engage sur les traces d'une mère inconnue : "Ton programme tient en deux mots, ma tendre Malaïka. Retrouver la trace de ta mère première, la femme sans visage, inaccessible à ce jour ... . Mieux qu'un pèlerinage, c'est l'appel du sang, la quête des origines, la grève vers laquelle tendent les vagues de tes jours » (150). Après avoir levé le voile sur son passé, la jeune fille serait donc en mesure de se défaire d'une identité opaque. Et pourtant la rencontre avec la 'mère première' se solde par un échec. Le rapport à l'Autre se traduit par une désunion des corps, rendant l'amalgame entre construction identitaire et impératif généalogique profondément caduque. Célestine enlace Maya dans une étreinte qui se veut affectueuse; toutefois la réponse physique de la jeune fille dénote un malaise qui traduit une effraction corporelle (172).

De même, le lieu des origines de Maya est loin de permettre une possible inscription spatiale, un rapport au lieu. Son voyage en France résulte plutôt en un détachement spatial qui attribue à Maya le statut d'étrangère. La France n'est pas terre d'accueil, mais plutôt lieu d'enfermement qui se livre tout comme les É-U d'Afrique à un repli identitaire. Dans cette perspective, le nomadisme qui a cours parmi les 'immigrés extra-africains' suppose certes un mouvement dans l'espace placé sous le signe d'une nécessité économique, mais nomadisme n'est pas nécessairement gage d'ouverture. Ici, la présence d' 'immigrés extra-africains' sur le territoire des É-U d'Afrique confronte plutôt deux structures d'enfermement. Une fois en France, la rencontre de Maya avec 'les autochtones' n'est que la confirmation et le renforcement de la pratique exclusive du repli identitaire. À l'image des États-Uniens d'Afrique qui mettent en œuvre une politique inhospitalière, l'hémisphère Nord, et plus particulièrement la France, se caractérise par une « fierté d'être français » — fierté qui exclut en ce qu'elle donne sens à « la 
peur qu'ils ont les uns des autres ... exacerbée par l'ignorance profonde où ils sont, à gué, les uns par rapport aux autres » (159). Par conséquent, tout comme Maya se distinguait du 'nous' des États-Uniens d'Afrique, elle se trouve à nouveau en marge du 'ils' des Euraméricains.

L'errance ne peut donc pas être réduite à un retour aux origines, surtout lorsque celui-ci engage un enracinement identitaire exclusif. En particulier, la prévalence d'origines totalisantes s'inscrit en parfaite opposition avec le mouvement engagé par Maya depuis le début du récit. Il ne s'agit pas de ne plus envisager l'errance comme possible stratégie esthétique et éthique pour une ré/conciliation des différences, mais plutôt de concevoir l'échec du voyage de Maya comme la remise en question du modèle d'opposition binaire qui lui met à la marge ce qui est (ou perçu comme) Autre, différent. D'ailleurs, Maya demeure une figure en mouvement qui poursuit son errance et retourne à Asmara dans la dernière partie du récit — un retour qui s'apparente plutôt à un détour, et permet d'esquisser en filigrane la mise en pratique de voies de ré/conciliation des différences culturelles.

La distinction qu'il s'agit d'élaborer entre le retour et le détour suggère d'assimiler le retour à une trajectoire linéaire, tandis que le détour désigne plutôt le processus d'éparpillement spatial et identitaire caractéristique de l'errance. Rappelons que dans Les Entretiens de Baton Rouge, Glissant évoque 'une pratique du détour' qui permet de mettre en texte l'errance lorsqu'il précise notamment: « c'est quitter, se départir du lieu qui vous a été donné, pour mieux le comprendre, ou pour mieux vivre avec» (109). Dans cette perspective, l'exploration des multiples niveaux de l'errance, à la fois mental, métaphorique et physique, rend possible la présence à soi et aux Autres de Maya. Le personnage devient dépositaire d'interactions renouvelées avec les particuliers en ce qu'elle est d'une part, « apaisée et réconciliée avec [elle-]même » et d'autre part, en mesure d'accroître sa présence aux Autres (183). Dans cette perspective, le voyage de Maya dans les territoires lointains s'articule tout d'abord autour d'une confrontation à une altérité exclusive et excluante, puis d'une reconnaissance et acceptation de la distance et de l'insaisissabilité de l'Autre participant enfin de l'ébauche d'une ouverture à l'altérité et ses diverses formes d'expression. Ouverture qui est portée dans le récit par le personnage seul de Malaika puisque « amour et commerce des corps » (188) sont mis en attente mais révèlent toutefois l'horizon d'espérance d'une interaction renouvelée avec l'inconnu. 


\section{Bibliographie}

Appiah, Anthony Kwame. Pour un nouveau cosmopolitisme. Paris : Odile Jacob, 2008.

Ashcroft, Bill, Gareth Griffiths, and Helen Tiffin. The Empire Writes Back: Theory and Practice in Post-Colonial Literatures (1989). New York: Routledge, 2002.

Ben Jelloun, Tahar. Hospitalité Française. Paris: Seuil, 1983.

Diop, Cheikh Mouhamadou. Fondements et Représentations Identitaires chez Ahmadou Kourouma, Tahar Ben Jelloun et Abdourahman Waberi. Paris: L'Harmattan, 2008.

Garane, Jeanne, ed. Discursive Geographies: Writing Space and Place in French. New York: Rodopi, 2005.

Glissant, Édouard. Poétique de la Relation (extraits). Paris: Gallimard, 1990.

---. Les Entretiens de Baton Rouge avec Alexandre Leupin. Paris: Gallimard, 2008.

Hassab-Charfi, Samia, and Sonia Zlitni-Fitouri, eds. Autour d'Édouard Glissant: Lectures, Épreuves, Extensions d'une Poétique de la Relation. Pessac: Presses Universitaires de Bordeaux, 2008.

Le Bris, Michel, and Jean Rouaud, dir. Pour une Littérature-Monde. Paris: Gallimard, 2007.

Loingsigh, Aedín Ní. « Destination Littérature-Monde? Travel and Language in Aux États-Unis d'Afrique. » Australian Journal of French Studies, vol. 48, no. 1, 2011, pp. 74-87.

Mabanckou, Alain. « Portraits d'Écrivains (9). Dix questions à Abdourahman Waberi. » Congo Page. http://www.congopage.com/Portraits-d-ecrivains9-Dix. Juin 2006, consulté le 5 août 2019.

Mangeon, Anthony. La pensée noire et l'Occident : de la bibliothèque coloniale à Barack Obama. Cabris : Éditions Sulliver, 2010.

Mbembe, Achille. On the Postcolony. Berkeley: University of California Press, 2001.

Onyeoziri, Gloria Nne. Shaken Wisdom: Irony and Meaning in Postcolonial African Fiction. Charlottesville: University of Virginia Press, 2011.

« Pour une 'Littérature-Monde' en Français. » Le Monde.

https://www.lemonde.fr/livres/article/2007/03/15/des-ecrivains-plaidentpour-un-roman-en-francais-ouvert-sur-le-monde_883572_3260.html). 15 mars 2007, consulté le 5 août 2019.

Prabhu, Anjali. « Aux États-Unis d'Afrique de Abdourahman Waberi: Narration Dialogique ou Dialectique?». Euvres \& Critiques, vol. XXXVI, no. 2, 2011, pp. 79-92.

Said, Edward. Orientalism (1978). London: Penguin, 2012.

---. Culture and Imperialism. New York: Vintage, 1993.

Waberi, Abdourahman. Aux États-Unis d'Afrique. Arles: Babel, 2006. 